\title{
GODeM: A Graphical Ontology Design Methodology
}

\author{
Rafaela Blanca Silva-López ${ }^{1}$, Mónica Silva-López ${ }^{1}$, Maricela Bravo ${ }^{1}$, \\ Iris Iddaly Méndez-Gurrola ${ }^{1}$, and Victor Germán Sánchez Arias ${ }^{2}$ \\ ${ }^{1}$ Universidad Autónoma Metropolitana, Distrito Federal, \\ Mexico \\ ${ }^{2}$ Universidad Nacional Autónoma de México, Distrito Federal, \\ Mexico \\ \{rbsl,misl,mcbc\}@correo.azc.uam.mx, iddalym@yahoo.com.mx, \\ victor_sanchez@cuaed.unam.mx
}

\begin{abstract}
In this paper we present a simple and didactic methodology to design an ontology for educational purposes. This methodology considers and incorporates the steps of the most outstanding methodologies for ontology design. Some of the reported methodologies specialize on the analysis of the knowledge domain, others in the formality of some of the language used to define it, others in the evaluation and documentation. Graphical Design Methodology (GODeM) is based on the methodological principles reported by Noy \& McGuiness, the OntoDesign Methontology, Entreprise Ontology, Toronto Virtual Enterprise and graphical notations. GODeM methodology is used for designing an ontological model which main objective is to personalize learning activities consistent with the student's learning profile.
\end{abstract}

Keywords: Ontology design, ontology visualization, methodology for building ontologies, ontological model.

\section{Introduction}

Ontology design and construction is an arduous task which requires the organization of knowledge into standardized models, in order to categorize the information so it can be automatically processed by computers. The creation of intelligent systems requires ontological models, so it is necessary to have a simple and didactic methodology that facilitates the design and implementation of ontologies using a graphical notation that promotes the standardization of the graphical representation of ontology designs.

\subsection{Conceptual Framework}

The word ontology comes from the Greek roots ontos (being) and logos (treated). The German philosophers used to differentiate the study of being the study of the types of beings in the natural sciences. 
The term ontology is adopted in Artificial Intelligence as a mechanism to share and reuse knowledge. Guarino defines the concept of ontology as a device constituted by a specific vocabulary that describes a knowledge domain, integrating a set of rules that specify such vocabulary [1][2]. While McGuinness defined ontology as the formal explicit description of concepts in a domain, including its properties and constraints that exist [3]. Although there are many different definitions of ontology, one of the most accepted is that of Thomas Gruber, who defined ontology as "a formal explicit specification of a shared conceptualization " [4].

Where conceptualization means that any ontology defines an abstract model ( attributes, values and relationships) of the knowledge domain it represents. Explicit specification means that an ontology represents the description and representation of concepts in an unambiguous way. Formal, states that an ontology must be represented formally, so it can be reused, shared and understood by any agent or machine. Finally, the term shared concerns formal and explicit representation of concepts that have been agreed by a team of knowledge domain experts. It can be concluded that the main reason to build an ontology is to share information and reuse knowledge we have about a specific domain.

Based on the proposals of Gruber [4] and McGuinness [2], in this work, the term ontology refers to an explicit formal specification of concepts in a domain of shared knowledge, including their properties and constraints.

\subsection{Components of an Ontology}

From the point of view of engineering, an ontology is a device constituted by a specific vocabulary, used to describe a certain reality, includes a set of assumptions that determine the meaning of the vocabulary. Thus, components of an ontology is a hierarchy of classes with attributes and relationships, a semantic network that represents a set of interrelated instances, a set of axioms about classes and/or instances, and a set of rules inference. The literature shows that the components of a domain ontology depend on the interest and needs of the developer. They are based ontologies components proposed by Sowa, Noy \& McGuinness and Farquhar [5-7].

\section{Overview of Methodologies for Ontology Design}

In this section we describe a set of related concepts concerned with methodologies for ontology design.

- Methodology: A set of methods and techniques that guarantee the quality of the results of an ontology design process.

- Method: ordered set of steps to develop a product.

- Technique: A procedure to achieve a goal [8]. Therefore, the methodology provides a framework to build an ontology for the domain of knowledge.

- Knowledge Engineering is the discipline derived from Artificial Intelligence responsible for the design and development of knowledge-based systems or expert 
systems. It relies on instructional methodologies, ICT and Computer Science to represent knowledge in a domain of knowledge.

Ontology developers or engineers frequently search for a methodology to appropriately design an ontology; however, many variables are present and becomes a difficult task in many cases. There is currently no standard method for building ontologies, each methodology includes different steps and different considerations. Some methods used for the design of ontologies are listed in table 1.

Table 1. Ontology design methods.

\begin{tabular}{|c|c|c|}
\hline Authors & Year & Methodology \\
\hline $\begin{array}{l}\text { University of Stanford } \\
\text { Natalya F. Noy and Deborah L. McGuinness } \\
\text { [6] }\end{array}$ & 2000 & $\begin{array}{l}\text { Ontology Development 101: A } \\
\text { Guide to Creating Your First } \\
\text { Ontolog }\end{array}$ \\
\hline Uschold and King [21] & 1995 & Enterprise Ontology \\
\hline Grüninger and Fox [19] & 1995 & $\begin{array}{l}\text { TOVE (Toronto Virtual } \\
\text { Enterprise) }\end{array}$ \\
\hline M. Uschold and M. Grüninger [20][22] & 1996 & $\begin{array}{l}\text { ONTOLOGIES: Principles, } \\
\text { Methods and Applications }\end{array}$ \\
\hline $\begin{array}{l}\text { Group of Ontological Engineering of the } \\
\text { Polytechnic University of Madrid [12] }\end{array}$ & 1997 & Methontology \\
\hline
\end{tabular}

Methontology is a mature methodology, requires the integration of processes, in addition to requiring more detailed activities involved. Uschold, King, Grüninger, and Fox's methodologies do not describe activities, processes, techniques, or life cycle. Noy and McGuinness do not consider the documentation of the ontology.

\subsection{Ontology Development Methodology}

A methodology developed at Stanford University, proposed by Noy and McGuinness in [6]. It addresses the important aspects to be taken into account and suggests a method for ontology development. It proposes an iterative approach, adds details in each iteration, taking modeling decisions throughout the process. This methodology proposes the following steps for the design of ontologies: 1) Determine the domain and scope of the ontology [9]; 2) Consider reusing existing ontologies; 3) Enumerate important terms in the ontology; 4) Define the classes and their hierarchy [10]; 5) Define the properties and slots of classes; 6) Define the facets or restrictions on the properties and slots; and 7) Create instances.

This methodology focuses on understanding the knowledge domain for proper design of the ontology, describes the basic elements of the ontology, considers its implementation and validation to populate with data. It is the simplest methodology; however, it does not consider points such as the evaluation and documentation of the ontology. 


\subsection{Methontology}

Methodology developed at the Polytechnic University of Madrid. Proposes an evolutionary prototyping process and procedure for the construction of an ontology [11-15]. Methonthology defines seven steps for the design of ontologies: 1) specification. [16]; 2) conceptualization; 3) acquisition; 4) integration; 5) implementation; 6) evaluation; and 7) documentation.

This methodology has a high degree of analysis to understand the domain of knowledge to model, constantly involves the knowledge domain experts, to collect information before the design of the ontology. We must be careful not to fall into an over- analysis which takes a long time. Methontology proposes the use of intermediate representations that facilitate the understanding of domain experts and formal languages. It has a strong foundation in knowledge engineering methodologies and software development process.

\subsection{Enterprise Ontology Methodology}

Enterprise Ontology is used as the basis of other proposed methods. The methodology includes four steps and provides design recommendations that should be present during the design and construction of the ontology [10], [17-18]: 1) Identify the purpose and scope of the ontology; 2) Building ontology (identify knowledge, encode knowledge and Integrate knowledge); 3) Evaluate the ontology; and 4) Document the ontology.

This methodology has only four steps, which greatly simplifies the work and proposes to encode the ontology in a formal language. It raises the need to evaluate the ontology through competency questions similarly as in software requirements specification. All methods start with identifying the purpose of the ontology and understanding of domain knowledge.

\subsection{Gruninger and Fox Methodology}

Toronto Virtual Enterprise (TOVE), this methodology proposes a scenario-based process to describe the functionality of the ontology [19-22]. The steps that found this methodology are six: 1) Identify relevant scenarios; 2) Develop relevant questions in an informal (natural language); 3) Specifying ontology terminology; 4) Develop relevant questions formally; 5) Specify the axioms and theorems; and 6) Evaluate the ontology. The key points of this methodology are: identify queries, objects and predicates in the ontology. Apply a high degree of formality as they resort to logicalmathematical language for the formal description of the relevant questions of the axioms and theorems.

Methontology is the most mature methodology; however, it does not consider competency questions, and the instantiation of individuals requires the incorporation of restrictions on properties. Grüninger and Fox's does not consider the reuse of existing ontologies. The metodologies of Uschold-King's and Grüninger-Fox's not describe activities, processes, techniques, or lifecycle. Finally, none of the 
methodologies considers a graphical notation to represent the design of ontology clearly and simple way. It is therefore desirable to have a methodology that integrates these features.

\section{Graphical Ontology Design Methodology (GODeM)}

Guizzardi and Botti propose OntoUML in reprising the entity relationship model adapted to the modeling of ontologies, however, is unclear, and focuses on information representing each of the classes, its cardinality and relationship [23]. It is complex to represent all the features it has an ontology. Meanwhile, Ceccaroni and Kendall, propose a graphical environment for ontology development in which only make use of the UML class diagram, so there is no detail on the characteristics, properties and relationships of the various classes that make up the ontology [24].

Interactive Visualization of Large OWL Instance Sets, proposed by Liebig and Noppens, hierarchy diagrams used to represent the relationships between classes in the ontology, but does not include features, properties and detail of the relationships between classes. This type of diagram is informative, not graphically depicts all the features of an ontology [25].

Negru, Haag and Lohmann, have Unified Visual Notation for OWL Ontologies, which are used hierarchical diagrams using UML notation to represent the classes and their relationships to other classes. It does not include detailed information on the characteristics, properties and additional information relationships [26].

Furthermore, Lohmann, Negru, Haag, and Ertl, present the VOWL 2: UserOriented Visualization of Ontologies, which allows graphically represent an ontology. This proposal is very similar to ours, using symbols to represent classes, properties, relationships, direction of relationships, cardinality, relationship types (data properties and object properties) and some colors to represent different types of properties [27].

OntoDesign Graphics can represent relationships between classes in the ontology as well as its characteristics and properties. Adds a graphical notation to integrate multiple ontologies and we only establish relationships between classes, but between ontologies. We use ovals instead of circles which gives more clarity to the graphical representation.

After analyzing the various methodologies for ontology design, we propose a methodology based on the principles of methodologies of Noy \& McGuiness, Methontology, Grüninger Fox's, Enterprise Ontology and OntoDesign Graphics. The proposed methodology integrates the simplicity and detail offered by Noy \& McGuiness methodology to understand the domain of knowledge and make a good design, at the same time, it integrates a graphical notation formal language that allows to visualize as a whole ontology design through OntoDesign Graphics. Finally incorporates the steppes of validation and documentation as required Methontology.

OntoDesign Graphics is a proposal for a notation that can represent grafically designing an ontology, visually in a single diagram can identify all the elements of the ontology, such as classes, class hierarchy, properties, relations between classes, 
characteristics of properties, among others. Enrich documentation and facilitates the understanding of the design to other users [28].

OntoDesign Graphics integration having aim to have graphical notation that allows standardizing ontologies designs for clarity, as with the use of UML notation. For example, authors such as Rezgui, Mhiri, Ghédira, Ali,Tawil, Jahankhani, Yarandi, Mencke, Dumke, Bouhdidi, Ghailani, and Fennan [29-32], show a great diversity in the graphical representation of ontologies designs proposed which complicates interpretation between one notation and another.

We propose a methodology: Graphical Ontology Design Methodology (GODEM). The main goals of this methodology are: simplicity and didactic, used for teaching and educational. Facilitates the first ontology design a simple yet detailed guide you to achieve your goal.

GODeM the methodology is comprised of the following steps:

1. Specify the domain of knowledge and scope of the ontology.

(a) Analyze the key elements involved in the domain of knowledge. Conduct interviews with experts in the domain of knowledge.

(b) Prepare diagrams showing the relationships and characteristics of the key elements of the knowledge domain visually. Its aim is to facilitate feedback with expert domain knowledge.

2. Identification of requirements ontology.

(a) Define the relevant questions that must be answered by ontology, also known as competency questions.

3. Validation of the possibility of using existing ontologies or metadata.

(a) Browse and search in different repositories of ontologies related to the domain of knowledge that is addressed, to identify whether it is possible to reuse an ontology.

4. Ontological model design.

(a) List important terms of ontology to develop a glossary of terms.

(b) Define the classes and their hierarchy.

(c) Define the properties or attributes of classes.

(d) Define restrictions on properties (data type, cardinality, domain and range).

(e) Elaborate design ontology with OntoDesign Graphics notation.

(f) Populate the preliminar ontology design to detect and correct errors during design. In case of errors repeat the activities listed in subsection.

5. Implementation of the ontological model.

(a) Select the language to use (OWL).

(b) Select the tool for implementation (Protégé).

6. Populate classes.

(a) Create instances or individuals populate the ontology with real world data.

7. Evaluation

(a) Verification of ontology. Apply the rules established by [6]:

(i) There are multiple solutions to model a domain. The best solution is given during the process depending on the purpose of the ontology and its applications. 
(ii) The development of an ontology is an iterative incremental process.

(iii) Ontology classes are objects in the domain of knowledge and relationships are associated with verbs that are identified in the relevant questions that must be answered ontology.

(b) Validation of the ontology.

(i) Determine if the ontology answers the questions of competence.

8. Document the ontology.

(a) Document the steps taken during the design and implementation of ontology to share and reuse.

\section{Case Study}

The methodology used for the design of an educational ontological model in order to personalize learning activities to enhance learning and thus school passing rates. It is intended that the ontological model various cognitive theories applied to determine the student's learning profile, allowing you select learning activities that will improve their motivation and learning activities that promote the development of cognitive skills. Its aim is customizing learning activities from cognitive skills that develop students want.

To set the domain of knowledge and scope of the ontology, will discuss the key elements involved in the domain of knowledge and draw diagrams showing the relationships between the key elements and features appears. The diagrams facilitate communication with the domain expert knowledge, are a simple feedback and enabling understanding of the knowledge domain in question.

\subsection{Analysis of the Key Elements Involved in the Domain of Knowledge}

The key elements involved in the domain of knowledge are: personalization learning activities, the learning profile of the student, the course, and the student's general data.

To identify the requirements of the ontology is necessary to develop a list of relevant questions that must be answered by the ontology.

For the domain of knowledge we found the following competency questions:

What is the domain of the ontology? The ontology focuses on the educational domain, specifically in the teaching-learning process. Particularly focusing on the customization of the assessment.

What is the use of the ontology? To customize assessment activities in accordance with the profile of an individual's learning and mastering knowledge of teaching a course. The experimental evaluation case that will be used is the course "Structured Programming" with engineering students.

Who will use the ontology? Ontology users are students and teachers. For the particular case of fieldwork, students will apply Engineering with massive semi - face courses, the Structured Programming course. 


\subsection{Competency Questions}

The following list is the set of questions that the ontology should answer:

1. What are the cognitive types for a cognitive theory $X$ ?

2. What characteristics does a guy cognitive $\mathrm{Z}$ for a cognitive theory $\mathrm{Y}$ ?

3. What is the student's learning profile $X$ ?

4. What are the cognitive characteristics that a student $\mathrm{X}$ has?

5. What learning activities are recommended for the course that requires developing the cognitive ability $\mathrm{Y}$ ?

6. What learning activities are recommended for learning profile $X$ ?

7. What kind of tool should be recommended to perform an activity that develops a cognitive skill Y?

8. What assessment activity should make a student with learning profile for the course W X Y module?

9. What is the recommended learning path for a student with learning profile W?

\subsection{Identification of Key Concepts and Axiomatization of the Ontology}

The development of the conceptual model of ontology starts with the list of key terms that relate to the field of knowledge that is addressed and worked the glossary of terms shown in table 2 . The axiomatization establishes necessary and sufficient restrictions for class properties. It is important to add annotations to the classes for a formal design documentation. Now, to axiomatize must define constraints on the properties, therefore specifies the data type, type of cardinality, as well as its domain and range. The axiomatization of classes of Profiles ontology is shown in table 3.

Table 2. Glossary of terms.

\begin{tabular}{ll}
\hline \multicolumn{1}{c}{ Concept } & \multicolumn{1}{c}{ Description } \\
\hline \hline $\begin{array}{l}\text { Student } \\
\text { Learning profile }\end{array}$ & $\begin{array}{l}\text { Individual requires a personalized learning path. } \\
\text { Characteristics that differentiate people and for determining how to learn } \\
\text { and think. } \\
\text { Thematic content of the discipline to be taught. } \\
\text { Course }\end{array}$ \\
$\begin{array}{l}\text { Mvaluation } \\
\text { Module }\end{array}$ & $\begin{array}{l}\text { Section of the course addresses a specific topic as part of the course. } \\
\text { Multimedia }\end{array}$ \\
$\begin{array}{l}\text { Mducational } \\
\text { Resource }\end{array}$ & videos, recorded lectures, electronic books, among others. \\
Cognitive Ability & Skills to be developed by the student to complete course. \\
Evaluation Type & Characteristics that determine the type assessment student knowledge. \\
Activity & Learning activities performed by the student. \\
Cognitive Style & Determine the characteristics that identify a learning profile. \\
Learning Path & The system offers to personalize the activities of student per module.
\end{tabular}


Table 3. Axiomatization of classes of Profiles ontology.

\begin{tabular}{llll}
\hline \multicolumn{1}{c}{ Class } & \multicolumn{1}{c}{ Property } & Data type & \multicolumn{1}{c}{ Cardinality } \\
\hline \hline CognitiveStyle & Description & String & $\equiv 1$ \\
& Author & Symbol & $\equiv 1$ \\
& URLTest & String & $0 \geq \leq 1$ \\
CognitiveType & Name & String & $\equiv 1$ \\
CognitiveFeature & Description & String & $\equiv 1$ \\
\hline
\end{tabular}

\subsection{Design and Implementation of the Ontology}

The design of the ontological model consists of 5 ontologies: Profiles, Students, Courses, AssessmentActivities and LearningPath. This ontological model stores the profile of student learning, and from cognitive skills identified, the activities are customized in order to foster the development of skills in line with the objectives of each course unit. OWL DL is the standard description formal language to specify ontologies, the reasoning is Pellet and the tool used to implement the ontology is Protégé as shown in figure 1. The ontology was modeled with OntoDesign Graphics, and it's shown in the figure 2 .

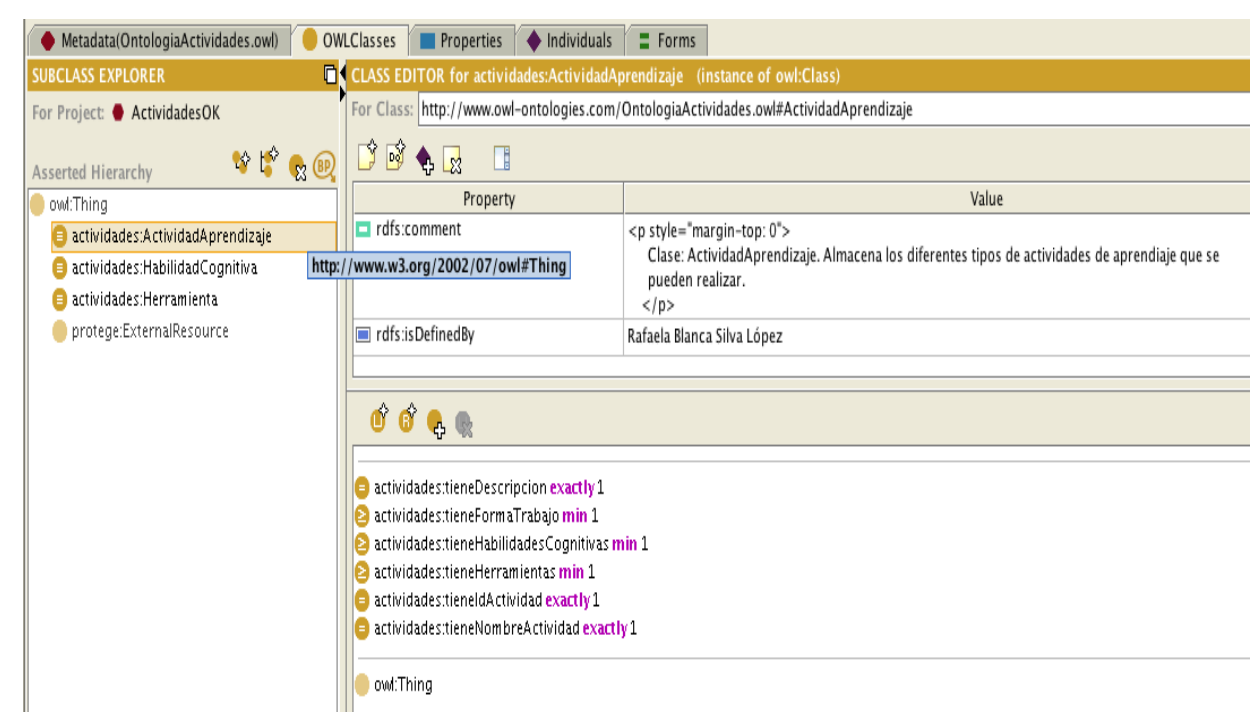

Fig. 1. Implementing the ontology in Protégé.

\section{Conclusions}

In this paper, we have described the Graphical Ontology Design Methodology (GODeM). One of the most important things is the incorporation of use OntoDesign Graphics notation. 

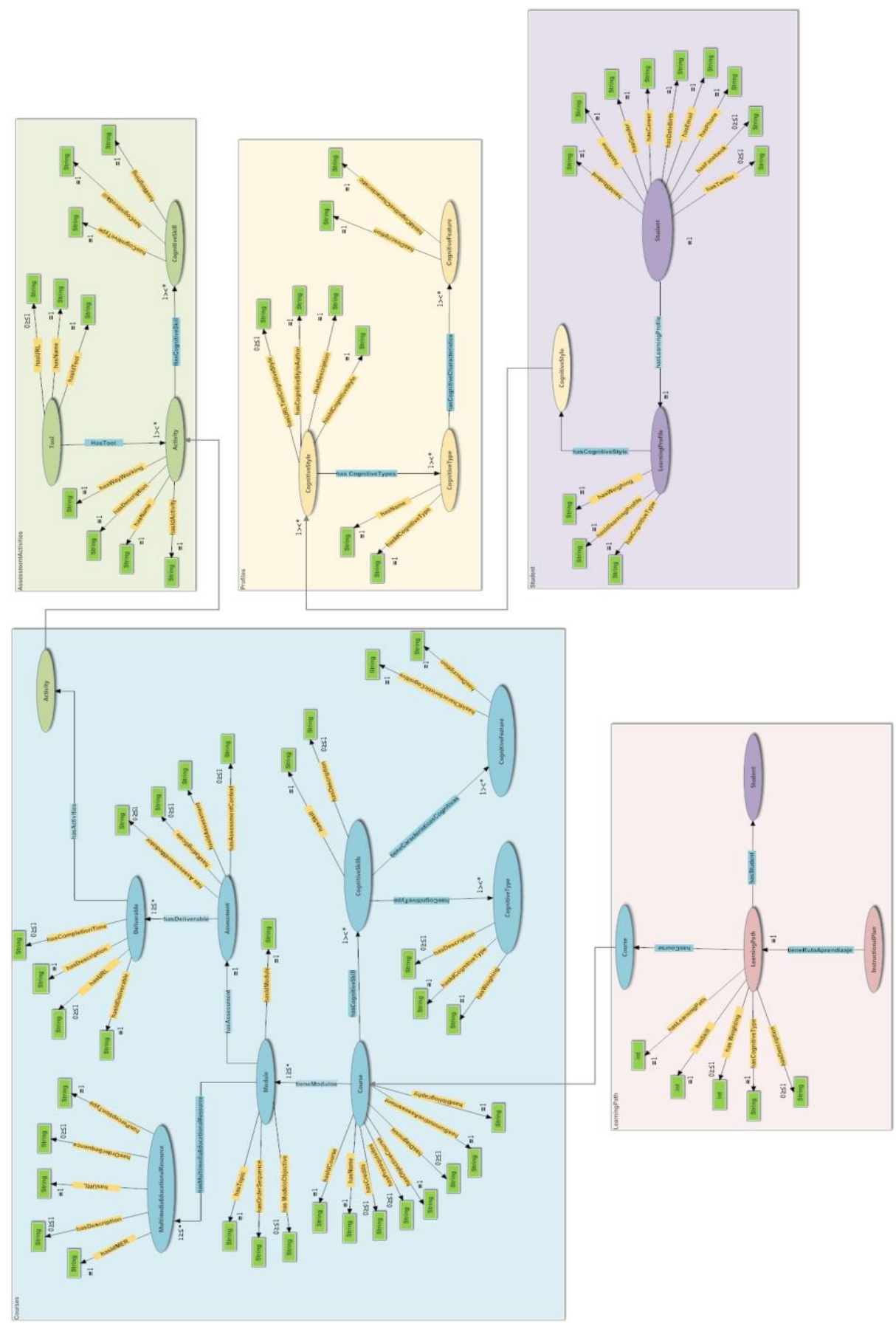

Fig. 2. Semantic relations between ontologies with OntoDesign Graphics. 
However, the ontology design is a creative processand, two ontologies designed by different people would be different. The potential applications of the GODeM is incorporating a graphical notation used in the design of ontologies to have an easier to reuse documentation. Finally, we can assess the quality of our ontology by using it in applications for which we designed it. The results generated determine adjustments that must be made.

Acknowledgements. This work is part of the research undertaken by Blanca Silva to obtain the PhD at UDG-Virtual, México, and it is supported by Universidad Autónoma Metropolitana. Also this work is part of the project PAPIIT UNAM IT100213.

\section{References}

1. Guarino, N.: Understanding, Building and Using Ontologies. International Journal of Human Computer Studies. pp. 293-310 (1997)

2. Guarino, N.: Formal Ontology and Information Systems. Proceedings of the 1st International Conference on Formal Ontologies in Information Systems, FOIS'98, pp. 315. IOS Press (1998)

3. McGuinness, D.L: Ontologies Come of Age. Fensel, D., Hendler, J., Lieberman, H., Wahlster, W. Editors. The Semantic Web: Why, What, and How, MIT Press, (2003)

4. Gruber,T.R.: A Translation Approach to Portable Ontologies. Knowledge Acquisition, pp. 199-220 (1993)

5. Sowa, J.F.: Knowledge Representation: Logical, Philosophical, and Computational Foundations. Pacific Grove, CA: Brooks Cole Publishing Co (2000)

6. Noy, N.F., McGuinness, D.L.: Ontology Development 101: A Guide to Creating Your First Ontology. Stanford Knowledge Systems Laboratory Technical Report KSL-01-05 and Stanford Medical Informatics Technical Report SMI-2001-0880 (2001)

7. Farquhar, A.: Ontolingua Tutorial. Knowledge Systems Lab. University of Stanford. [Online].

8. Ander-Egg, E.: Técnicas de investigación social para trabajadores sociales. Buenos Aires: El Cid Editor (1978)

9. Grüninger, M., Fox, M.S.: Methodology for the Design and Evaluation of Ontologies. In Proceedings of the Workshop on Basic Ontological Issues in Knowledge Sharing, IJCAI95 (1995)

10. Uschold, U., Grüninger, M.: Ontologies: Principles, Methods and Applications. Knowledge Engineering. Review, pp. 93-155 (1996)

11. Gómez-Pérez, A.: A Framework to Verify Knowledge Sharing Technology. Expert Systems with Application, pp. 519-529 (1996)

12. Fernández, M., Gómez-Pérez, A., Juristo, N.: METHONTOLOGY: From Ontological Art Towards Ontological Engineering. In Proceedings of AAAI97 Spring Symposium Series, Workshop on Ontological Engineering, pp. 33-40 (1997)

13. Gómez-Pérez, A.: Knowledge Sharing and Reuse. In J. Liebowitz (Ed.) Handbook of Expert Systems. CRC (1998)

14. Fernández, M.: Overview of Methodologies for Building Ontologies. In V. R Benjamins (Ed.) Proceedings of IJCAI99 Workshop on Ontologies and Problem-Solving Methods: Lessons Learned and Future Trends, Vol. 18, CEUR Publications (1999) 
15. Fernández, M., Gómez-Pérez, A., Pazos-Sierra, A., Pazos-Sierra, J.: Building a Chemical Ontology Using Methontology and the Ontology Design Environment. IEEE Intelligent Systems, pp. 37-46 (1999)

16. García Peñalvo, F.J.: Web Semántica y Ontologías. (2005) [Online]. Available: http://zarza.usal.es/ fgarcia/doctorado/iuce/WSemantica.pdf

17. Uschold, M., King, M.: Towards a Methodology for Building Ontologies. In Proceedings of IJCAI95's Workshop on Basic Ontological Issues in Knowledge Sharing (1995)

18. Uschold, M.: Building Ontologies: Towards a Unified Methodology. In Proceedings of 16th Annual Conference of the British Computer Society Specialist Group on Expert Systems (1996)

19. Grüninger, M., Fox, M.S.: The Design and Evaluation of Ontologies for Enterprise Engineering. In Proceedings of the Workshop on Implemented Ontologies, European Conference on Artificial Intelligence (1994)

20. Grüninger, M., Fox, M.S. The Role of Competency Questions in Enterprise Engineering. In Proceedings of the IFIP WG5.7 Workshop on Benchmarking-Theory and Practice. (1994)

21. Grüninger, M., Fox, M.S.: The Logic of Enterprise Modelling. In Brown, J., O'Sullivan, D. (Eds.), Reengineering the Enterprise, pp. 83-98, Chapman and Hall (1995)

22. Grüninger, M.: Designing and Evaluating Generic Ontologies. In Proceedings of the 12th European Conference of Artificial Intelligence, pp. 53-65 (1996)

23. Botti, A., Guizzardi, G.: A Model-Based Tool for Conceptual Modeling and Domain Ontology Engineering in OntoUML, Springer-Verlag Berlin Heidelberg, pp. 528-539 (2009)

24. Ceccaroni, L., Kendall, E.: A Graphical Environment for Ontology Development. ACM 158113-683-8/03/0007, pp. 958-959 (2003)

25. Noppens, O., Liebig, T.: Interactive Visualization of Large OWL Instance Sets. In Proceedings of the Third Int. Semantic Web User Interaction Workshop, 2006. Athens, GA, USA (2006)

26. Negru, S., Haag, F., Lohmann, S.: Towards a Unified Visual Notation for OWL Ontologies: Insights from a Comparative User Study. In Proceedings of the 9th International Conference on Semantic Systems, New York, NY, USA: ACM (2013)

27. Lohmann, S., Negru, S., Haag, F., Ertl. T.: VOWL 2: User-Oriented Visualization of Ontologies. EKAW, (2014)

28. Silva-López, R.B., Silva-López, M., Méndez-Gurrola, I.I., Bravo, M.: Onto Design Graphics (ODG): A Graphical Notation to Standardize Ontology, MICAI 2014, Part I, LNAI 8856, Springer International Publishing Switzerland, pp. 443-452 (2014)

29. Rezgui, K., Mhiri, H., Ghédira, K.: An Ontology-based Profile for Learner Representation in Learning Networks. International Journal of Emerging Technologies in Learning, Vol. 9 (3), pp. 16-25 (2014)

30. Seyed Ali, H., Abdel-Rahman, H.T, Jahankhani, H., Yarandi, M.: Towards an Ontological Learners' Modelling Approach for Personalised e-Learning. In International Journal of Emerging Technologies in Learning, Vol. 8(2), pp. 4-10 (2013)

31. El Bouhdidi, J., Ghailani, M., Fennan, A.: A Probabilistic Approach for the Generation of Learning Sessions Tailored to the Learning Styles of Learners. In International Journal of Emerging Technologies in Learning, Vol. 8(6), pp. 42-49 (2013)

32. Mencke, S., Dumke, R.: Didactical Ontologies. In International Journal of Emerging Technologies in Learning, Vol. 3(1), pp. 65-73 (2008) 